\title{
Research Article \\ Entire Blow-Up Solutions of Semilinear Elliptic Systems with Quadratic Gradient Terms
}

\author{
Yongju Yang ${ }^{1}$ and Xinguang Zhang ${ }^{2}$ \\ ${ }^{1}$ School of Mathematics and Statistics, Nanyang Normal University, Henan, Nanyang 473061, China \\ ${ }^{2}$ School of Mathematical and Informational Sciences, Yantai University, Shandong, Yantai 264005, China
}

Correspondence should be addressed to Xinguang Zhang, zxg123242@sina.com

Received 1 September 2012; Accepted 8 November 2012

Academic Editor: Yong Hong Wu

Copyright (C) 2012 Y. Yang and X. Zhang. This is an open access article distributed under the Creative Commons Attribution License, which permits unrestricted use, distribution, and reproduction in any medium, provided the original work is properly cited.

We study the existence of entire positive solutions for the semilinear elliptic system with quadratic gradient terms, $\Delta u_{i}+\left|\nabla u_{i}\right|^{2}=p_{i}(|x|) f_{i}\left(u_{1}, u_{2}, \ldots, u_{d}\right)$ for $i=1,2, \ldots, d$ on $R^{N}, N \geq 3$ and $d \in\{1,2,3, \ldots\}$. We establish the conditions on $p_{i}$ that ensure the existence of nonnegative radial solutions blowing up at infinity and also the conditions for bounded solutions on the entire space. The condition on $f_{i}$ is simple and different to the Keller-Osserman condition.

\section{Introduction}

We study the existence of entire blow-up positive solutions of the following elliptic system with quadratic gradient terms:

$$
\begin{gathered}
\Delta u_{1}+\left|\nabla u_{1}\right|^{2}=p_{1}(|x|) f_{1}\left(u_{1}, u_{2}, \ldots, u_{d}\right), \quad x \in R^{N}, \\
\vdots \\
\vdots \\
\Delta u_{d}+\left|\nabla u_{d}\right|^{2}=p_{d}(|x|) f_{d}\left(u_{1}, u_{2}, \ldots, u_{d}\right), \quad x \in R^{N},
\end{gathered}
$$

where $d \geq 1, N \geq 3, p_{i}(i=1,2, \ldots, d)$ are $c$-positive functions and $f_{i}:[0, \infty)^{d} \rightarrow[0, \infty)$ are nonnegative, continuous, and nondecreasing functions for each variable. 
For convenience we recall the definitions about $c$-positive functions and entire blowup positive solutions.

(i) A function $p$ is $c$-positive (or circumferentially positive) in a domain $\Omega \subseteq R^{N}$ if $p$ is nonnegative on $\Omega$ and satisfies the following condition: if $x_{0} \in \Omega$ and $p\left(x_{0}\right)=0$, then there exists a domain $\Omega_{0}$ such that $x_{0} \in \Omega_{0} \subset \Omega$ and $p(x)>0$ for all $x \in \partial \Omega_{0}$.

(ii) A solution $\left(u_{1}, u_{2}, \ldots, u_{d}\right)$ of the system (1.1) is called an entire blow-up solution (or explosive solution) if it is a classical solution of the above problem on $R^{N}$ and $u_{i}(x) \rightarrow \infty,(i=1,2, \ldots, d)$ as $|x| \rightarrow \infty$.

Existence and nonexistence of blow-up solutions of semilinear elliptic equations and systems have received much attention worldwide. Bieberbach [1] is the first to study blow-up solutions to the semilinear elliptic problem

$$
\Delta u=f(u), \quad x \in \Omega,
$$

where $f(u)=e^{u}$. Following Bieberbach's work, many authors have studied related problems for single equations and systems. In 1957, Keller [2] and Osserman [3] established the necessary and sufficient conditions for the existence of solutions to (1.2) on bounded domains in $R^{n}$. They showed that blow-up solutions exist on $\Omega$ if and only if $f$ satisfies the following Keller-Osserman condition:

$$
\int_{1}^{+\infty}\left[\int_{0}^{t} f(s) d s\right]^{-1 / 2} d t<+\infty
$$

Bandle and Marcus [4] later examined the equation

$$
\Delta u=p(x) f(u)
$$

with $f$ is nondecreasing on $[0,+\infty)$ and proved the existence of positive blow-up solutions under the condition that the function $f$ satisfies the Keller-Osserman condition (1.3) and $p$ is continuous and strictly positive on $\bar{\Omega}$. Lair [5] showed that the results also hold for (1.4) when $p$ is allowed to vanish on a large part of $\Omega$, including its boundary. In addition, many authors have examined some more specific forms of (1.4). The equation

$$
\Delta u=p(x) u^{r}
$$

has been of particular interest. Cheng and $\mathrm{Ni}$ [6] considered the superlinear case $\gamma>1$ and proved that for this case (1.5) has blow-up solutions on bounded domains provided $p$ is strictly positive on $\partial \Omega$. Lair and Wood [7] generalized this to allow $p$ to vanish on some portions of $\Omega$ including its boundary and also showed the existence of an entire blow-up solution to (1.5) provided that

$$
\int_{0}^{+\infty} r \max _{|x|=r} p(r) d r<+\infty
$$

Obviously, condition (1.6) is weaker than the requirements in [6]. 
if

In [8], Lair and Wood proved that (1.5) has entire blow-up radial solutions if and only

$$
\int_{0}^{+\infty} r p(r) d r<+\infty
$$

They also demonstrated that for a bounded domain $\Omega,(1.5)$ has no positive blow-up solution when $p$ is continuous in $\Omega$. In addition, they proved that nonnegative, entire bounded solutions do not exist for (1.5) if

$$
\int_{0}^{+\infty} r \min _{|x|=r} p(r) d r=+\infty
$$

Although semilinear elliptic systems are the natural extension of single equations in many areas of applications, the results and methods for the study of single equations are often not applicable to the systems of equations. Recently, Lair and Wood [9] studied the existence of entire positive solutions of the system

$$
\begin{array}{ll}
\Delta u=p_{1}(|x|) v^{\alpha}, & x \in R^{N}, \\
\Delta v=p_{2}(|x|) u^{\beta}, & x \in R^{N} .
\end{array}
$$

In the sublinear case $0<\alpha \leq \beta \leq 1$, the authors proved that provided that the nonnegative functions $p$ and $q$ are continuous, $c$-positive, and satisfy the fast decay conditions

$$
\int_{0}^{\infty} t p_{i}(t) d t<\infty, \quad i=1,2
$$

then the entire positive solutions are bounded, while if $p$ and $q$ satisfy the slow decay conditions

$$
\int_{0}^{\infty} t p_{\mathrm{i}}(t) d t=\infty, \quad i=1,2
$$

then the entire positive solutions blow up. For the superlinear case $\alpha, \beta>1$, the fast decay conditions $(F)$ are required to hold. Later, Cîrstea and Rădulescu [10] improved the results of Lair and Wood [9] and proved that for $p, q \in C_{\mathrm{loc}}^{0, \alpha}\left(R^{N}\right)(0<\alpha<1)$, the following semilinear elliptic system

$$
\begin{array}{ll}
\Delta u=p_{1}(x) f(v), & x \in R^{N}, \\
\Delta v=p_{2}(x) g(u), & x \in R^{N}
\end{array}
$$

has entire solutions if $f$ and $g$ satisfy

$$
\lim _{t \rightarrow \infty} \frac{f(c g(t))}{t}=0
$$


for all $c>0$ and has solutions that are bounded when $(D)$ holds. Further, entire solutions exist and are blow-up when $(F)$ holds. An analogous condition was also employed by Ghergu and Rădulescu [11] to study the following elliptic system with gradient terms:

$$
\begin{aligned}
& \Delta u+|\nabla u|=p_{1}(|x|) f(v), \quad x \in \Omega, \\
& \Delta v+|\nabla v|=p_{2}(|x|) g(u), \quad x \in \Omega,
\end{aligned}
$$

where $\Omega$ is a bounded domain or the whole space. Peng and Song [12] also studied the existence of entire blow-up positive solutions of system (1.10) when the $c$-positive functions $p_{i}, i=1,2$ satisfy the decay conditions $(F)$. Peng and Song [12] also imposed on $f$ and $g$ the following Keller-Osserman conditions:

$$
\int_{1}^{\infty}\left[\int_{0}^{s} f(t) d t\right]^{-1 / 2} d s<\infty, \quad \int_{1}^{\infty}\left[\int_{0}^{s} g(t) d t\right]^{-1 / 2} d s<\infty
$$

and the convexity conditions

$$
\begin{gathered}
f(\lambda a+(1-\lambda) b) \leq \lambda f(a)+(1-\lambda) f(b), \quad \lambda \in(0,1), \\
g(\lambda a+(1-\lambda) b) \leq \lambda g(a)+(1-\lambda) g(b), \quad a, b \geq 0 .
\end{gathered}
$$

Both papers [6, 12] considered system (1.10) where the nonnegative functions $p_{i}(i=1,2) \in$ $C[0,+\infty)$ satisfy $(F)$ and the functions $f, g \in C[0,+\infty)$ are nondecreasing and satisfy the Keller-Osserman condition (1.13), and

$$
f(0)=g(0)=0, \quad f(s)>0, \quad g(s)>0, \quad \text { for } s>0 .
$$

Recently, Zhang and Liu [13] studied the following semilinear elliptic system with the magnitude of the gradient

$$
\begin{array}{ll}
\Delta u+|\nabla u|=p(|x|) f(u, v), & x \in R^{N}, \\
\Delta v+|\nabla v|=q(|x|) g(u, v), & x \in R^{N} .
\end{array}
$$

The results of nonexistence of entire positive solutions have been established if $f$ and $g$ are sublinear and $p$ and $q$ have fast decay at infinity, while if $f$ and $g$ satisfy some growth conditions at infinity, and $p, q$ are of slow decay or fast decay at infinity, then the system 
has infinitely many entire solutions, which are large or bounded. In [14], Covei studied the existence of solution of the following semilinear elliptic system:

$$
\begin{gathered}
\Delta u_{1}=p_{1}(x) f_{1}\left(u_{1}, u_{2}, \ldots, u_{d}\right), \quad x \in R^{N}, \\
\vdots \\
\vdots \\
\Delta u_{d}=p_{d}(x) f_{d}\left(u_{1}, u_{2}, \ldots, u_{d}\right), \quad x \in R^{N} .
\end{gathered}
$$

Under some conditions on $f_{i}, p_{i}$, the system (1.17) has a bounded positive entire solution based on successive approximation. Furthermore, a nonradially symmetric solution also was obtained by using a lower and upper solution method. For more complicated Schrödinger systems, some nice work had been done by Covei in [15-17] with single equations or a system with $\left(p_{1}, \ldots, p_{d}\right)$-Laplacian in $R^{N}$. For further results on relevant work on single equations and/or systems as well as methods for the study of blow-up solutions of differential equations, see $[8,18-32]$ and the references therein.

The authors in $[13,14]$ only studied the semilinear elliptic system with the magnitude of the gradient term or without the gradient term. For elliptic systems involving nonlinear quadratic gradient terms, no result has been obtained. Thus, motivated by [11-17], we study the more general systems case with indefinite number of equations involving a nonlinear quadratic gradient term. In our results, a simple condition (2.5) has been used instead of the Keller-Osserman condition (1.13) commonly used in previous results. The main results obtained are presented in Section 2 by Theorems 2.3 to 2.6, while the proofs of the theorems are given in Section 3.

\section{Main Results}

For convenience in presenting the results, we here define

$$
\begin{gathered}
P_{i}(\infty)=\lim _{r \rightarrow \infty} P_{i}(r), \quad P_{i}(r)=\int_{0}^{r} t^{1-N} \int_{0}^{t} s^{N-1} p_{i}(s) d s d t, \quad r \geq 0, i=1,2, \ldots, d, \\
F(\infty)=\lim _{r \rightarrow \infty} F(r), \quad F(r)=\int_{a}^{r} \frac{d s}{\sum_{i=1}^{d} s f_{i}(\ln s, \ln s, \ldots, \ln s)}, \quad r \geq a>1, \quad i=1,2, \ldots, d .
\end{gathered}
$$

Remark 2.1. For any $i \in\{1,2, \ldots, d\}$, since

$$
F^{\prime}(r)=\frac{1}{\sum_{i=1}^{d} r f_{i}(\ln r, \ln r, \ldots, \ln r)}>0, \quad \forall r>a,
$$

$F$ admits the inverse function $F^{-1}$ on $[0, F(+\infty))$. 
Lemma 2.2 (see $[8,23])$. The slow decay condition

$$
\int_{0}^{\infty} t p_{i}(t) d t=\infty, \quad i=1,2, \ldots, d
$$

holds if and only if $P_{i}(\infty)=\infty$.

The first result we obtained is the condition for nonexistence of entire positive blowup solution, which asserts that if both $f_{i}, i=1,2, \ldots, d$ are bounded, then problem (1.1) does not have positive entire blow-up solution as detailed by the following theorem.

Theorem 2.3. Suppose $f_{i}, i=1,2, \ldots, d$ satisfy

$$
\max \left\{\sup _{\sum_{i=1}^{d} u_{i} \geq 1} f_{1}\left(u_{1}, u_{2}, \ldots, u_{d}\right), \ldots, \sup _{\sum_{i=1}^{d} u_{i} \geq 1} f_{d}\left(u_{1}, u_{2}, \ldots, u_{d}\right)\right\}<+\infty,
$$

and each $p_{i}, i=1,2, \ldots, d$ satisfy the decay conditions $(F)$. Then problem (1.1) does not have positive entire blow-up solution.

The other main results we obtained are the conditions, respectively, for the existence of infinitely many positive entire blow-up solutions and infinitely many positive entire bounded solutions, which are summarized in the following three theorems.

Theorem 2.4. If there exists a constant $a>1$ such that

$$
\int_{a}^{\infty} \frac{d s}{\sum_{i=1}^{d} s f_{i}(\ln s, \ln s, \ldots, \ln s)}=\infty,
$$

then the system (1.1) has infinitely many classical positive entire solutions $\left(u_{1}, u_{2}, \ldots, u_{d}\right)$. If, in addition, $p_{i}, i=1,2, \ldots, d$ satisfy the decay conditions $(D)$, then all the positive entire solutions of (1.1) are blow-up. Moreover, if $p_{i}, i=1,2, \ldots, d$ satisfy the decay conditions $(F)$, then all the positive entire solutions of (1.1) are bounded.

Theorem 2.5. If there exists a constant $a>1$ such that

$$
\int_{a}^{\infty} \frac{d s}{\sum_{i=1}^{d} s f_{i}(\ln s, \ln s, \ldots, \ln s)}<\infty
$$

and $p_{i}, i=1,2, \ldots, d$ satisfy the decay conditions $(F)$ and, in addition, there exist $b_{i}>a, i=$ $1,2, \ldots, d$ such that

$$
\sum_{i=1}^{d} P_{i}(\infty)<F(\infty)-F\left(\sum_{i=1}^{d} b_{i}\right)
$$

then the system (1.1) has a positive radial bounded solution $\left(u_{1}, u_{2}, \ldots, u_{d}\right)$ satisfying

$$
b_{i}+b_{i} f_{i}\left(\ln b_{1}, \ln b_{2}, \ldots, \ln b_{d}\right) P_{i}(r) \leq u_{i}(r) \leq F^{-1}\left(F\left(\sum_{i=1}^{d} b_{i}\right)+\sum_{i=1}^{d} P_{i}(r)\right), \quad r \geq 0 .
$$




\section{Theorem 2.6.}

(i) If $p_{i}, i=1,2, \ldots, d$ satisfy the decay conditions $(D)$ and

$$
\lim _{s \rightarrow \infty} \sum_{i=1}^{d} f_{i}(\ln s, \ln s, \ldots, \ln s)=0
$$

then the system (1.1) has infinitely many positive entire blow-up solutions.

(ii) If $p_{i}, i=1,2, \ldots, d$ satisfy the decay conditions $(F)$ and

$$
\sup _{s \geq 0} \sum_{i=1}^{d} s f_{i}(\ln s, \ln s, \ldots, \ln s)<\infty,
$$

then the system (1.1) has infinitely many positive entire bounded solutions.

\section{Proofs of the Theorems}

Firstly, via the change of variables $\phi_{i}=e^{u_{i}}, i=1,2, \ldots, d$, we turn the system (1.1) to the following equivalent system with no gradient terms

$$
\begin{array}{cc}
\Delta \phi_{1}=p_{1}(|x|) \phi_{1} f_{1}\left(\ln \phi_{1}, \ln \phi_{2}, \ldots, \ln \phi_{d}\right), & x \in R^{N}, \\
\vdots & \\
\vdots & \\
\Delta \phi_{i}=p_{i}(|x|) \phi_{i} f_{i}\left(\ln \phi_{1}, \ln \phi_{2}, \ldots, \ln \phi_{d}\right), \quad x \in R^{N}, \\
\vdots & \\
\vdots & \\
\Delta \phi_{d}=p_{d}(|x|) \phi_{d} f_{d}\left(\ln \phi_{1}, \ln \phi_{2}, \ldots, \ln \phi_{d}\right), & x \in R^{N} .
\end{array}
$$

Thus we only need to consider system (3.1).

Proof of Theorem 2.3. We use proof by contradiction to testify. We suppose that the system (3.1) has the positive entire blow-up solution $\left(\phi_{1}, \phi_{2}, \ldots, \phi_{d}\right)$. Consider the spherical average of $\phi_{i}$ defined by

$$
\bar{\phi}_{i}(r)=\frac{1}{c_{N} r^{N-1}} \int_{|x|=r} \phi_{i}(x) d \sigma_{x}, \quad r \geq 0,
$$

where $c_{N}$ is the surface area of the unit sphere in $R^{N}$. Since $\phi_{i}$ are positive entire blow-up solutions, it follows that $\bar{\phi}_{i}$ are positive and $\lim _{r \rightarrow \infty} \bar{\phi}_{i}(r)=+\infty$. By the change of variable $x=r y$, we have

$$
\bar{\phi}_{i}(r)=\frac{1}{c_{N}} \int_{|y|=1} \phi_{i}(r y) d \sigma_{y}, \quad r \geq 0 .
$$


Then

$$
\bar{\phi}_{i}^{\prime}(r)=\frac{1}{c_{N}} \int_{|y|=1} \nabla \phi_{i}(r y) \cdot y d \sigma_{y}, \quad r \geq 0
$$

Thus by the divergence theorem and (3.4), we have

$$
\begin{aligned}
\bar{\phi}_{i}^{\prime}(r) & =\frac{r}{c_{N}} \int_{|y|<1} \Delta \phi_{i}(r y) d y=\frac{1}{c_{N} r^{N-1}} \int_{|x|<r} \Delta \phi_{i}(x) d x \\
& =\frac{1}{c_{N} r^{N-1}} \int_{0}^{r} d \rho \int_{|x|=\rho} \Delta \phi_{i}(x) d \sigma_{x}, \quad \forall r \geq 0 .
\end{aligned}
$$

From [33], it follows from (3.5) that

$$
\begin{aligned}
\bar{\phi}_{i}^{\prime \prime}(r) & =\frac{1}{c_{N} r^{N-1}} \int_{|x|=r} \Delta \phi_{i}(x) d \sigma_{x}-\frac{N-1}{c_{N} r^{N}} \int_{0}^{r} d \rho \int_{|x|=\rho} \Delta \phi_{i}(x) d \sigma_{x} \\
& =\frac{1}{c_{N} r^{N-1}} \int_{|x|=r} \Delta \phi_{i}(x) d \sigma_{x}-\frac{N-1}{r} \bar{\phi}_{i}^{\prime}(r), \quad \forall r \geq 0 .
\end{aligned}
$$

Set

$$
U_{i}(r)=\max _{0 \leq t \leq r} \bar{\phi}_{i}(t)
$$

Then, obviously, $U_{i}$ are positive and nondecreasing functions. Moreover $U_{i} \geq \bar{\phi}_{i}$ and $U_{i}(r) \rightarrow$ $+\infty$ as $r \rightarrow+\infty$. Note from (2.4) that there exists $M>0$ such that

$$
\max \left\{f_{1}\left(u_{1}, u_{2}, \ldots, u_{d}\right), \ldots, f_{d}\left(u_{1}, u_{2}, \ldots, u_{d}\right)\right\} \leq M, \quad u_{1}+u_{2}+\cdots+u_{d} \geq 0 .
$$

Now (3.6) and (3.8) lead to

$$
\begin{aligned}
\bar{\phi}_{i}^{\prime \prime}+\frac{N-1}{r} \bar{\phi}_{i}^{\prime} & \leq \frac{1}{c_{N} r^{N-1}} \int_{|x|=r} \Delta \phi_{i}(x) d \sigma_{x} \\
& =p_{i}(r) \frac{1}{c_{N} r^{N-1}} \int_{|x|=r} \phi_{i}(x) f_{i}\left(\ln \phi_{1}(x), \ln \phi_{2}(x), \ldots, \ln \phi_{d}(x)\right) d \sigma_{x} \\
& \leq M p_{i}(r) \frac{1}{c_{N} r^{N-1}} \int_{|x|=r}\left(1+\sum_{i=1}^{d} \phi_{i}(x)\right) d \sigma_{x}=M p_{i}(r)\left(1+\sum_{i=1}^{d} \bar{\phi}_{i}(r)\right) \\
& \leq M p_{i}(r)\left(1+U_{i}(r)\right),
\end{aligned}
$$

for all $r \geq 0$. It follows that

$$
\left(r^{N-1} \bar{\phi}_{i}^{\prime}\right)^{\prime} \leq M r^{N-1} p_{i}(r)\left(1+U_{i}(r)\right), \quad r \geq 0
$$


So, for all $r \geq r_{0} \geq 0$, we have

$$
\begin{aligned}
\bar{\phi}_{i}(r) & \leq \bar{\phi}_{i}\left(r_{0}\right)+M \int_{r_{0}}^{r} t^{1-N} \int_{0}^{t} s^{N-1} p_{i}(s)\left(1+U_{i}(s)\right) d s d t \\
& \leq \bar{\phi}_{i}\left(r_{0}\right)+M \int_{r_{0}}^{r} t^{1-N}(1+U(t)) \int_{0}^{t} s^{N-1} p(s) d s d t \\
& \leq \bar{\phi}_{i}\left(r_{0}\right)+M\left(1+U_{i}(r)\right) \int_{r_{0}}^{r} t^{1-N} \int_{0}^{t} s^{N-1} p_{i}(s) d s d t \\
& \leq \bar{\phi}_{i}\left(r_{0}\right)+M\left(1+U_{i}(r)\right) \int_{r_{0}}^{r} t^{1-N} \int_{0}^{t} s^{N-1} p_{i}(s) d s d t \\
& \leq \bar{\phi}_{i}\left(r_{0}\right)+\frac{M\left(1+U_{i}(r)\right)}{N-2}\left(\int_{r_{0}}^{r} t p_{i}(t) d t-\frac{1}{r_{0}^{N-1}} \int_{r_{0}}^{r} t^{N-1} p_{i}(t) d t\right) \\
& \leq \bar{\phi}_{i}\left(r_{0}\right)+\frac{M\left(1+U_{i}(r)\right)}{N-2} \int_{r_{0}}^{r} t p_{i}(t) d t .
\end{aligned}
$$

Note that because of $(F)$, we can choose $r_{0}>0$ sufficiently large such that

$$
\max \left\{\int_{r_{0}}^{\infty} r p_{1}(r) d r, \ldots, \int_{r_{0}}^{\infty} r p_{d}(r) d r\right\}<\frac{N-2}{4 M} .
$$

Since $\lim _{r \rightarrow \infty} \bar{\phi}_{i}(t)=\infty$, it follows that we can find $r_{1} \geq r_{0}$ such that

$$
\bar{U}_{i}(r)=\max _{r_{0} \leq t \leq r} \bar{\phi}_{i}(t), \quad \forall r \geq r_{1} .
$$

Thus (3.11) and (3.13) yield

$$
\bar{U}_{i}(r) \leq \bar{\phi}_{i}\left(r_{0}\right)+\frac{M\left(1+\bar{U}_{i}(r)\right)}{N-2} \int_{r_{0}}^{r} t p_{i}(t) d t, \quad \forall r \geq r_{1} .
$$

By (3.12), we have

$$
\bar{U}_{i}(r) \leq \bar{\phi}_{i}\left(r_{0}\right)+\frac{1+\bar{U}_{i}(r)}{4}, \quad \forall r \geq r_{1}
$$

that is,

$$
\bar{U}_{i}(r) \leq C_{i}+\frac{\bar{U}_{i}(r)}{4}, \quad \forall r \geq r_{1}
$$

where $C_{i}=(1 / 4)+\bar{\phi}_{i}\left(r_{0}\right)>0$, which implies

$$
\sum_{i=1}^{d} \bar{U}_{i}(r) \leq \frac{4}{3} \sum_{i=1}^{d} C_{i}, \quad \forall r \geq r_{1}
$$


The inequality (3.17) means that $\bar{U}_{i}$ are bounded and so $\bar{\phi}_{i}$ are bounded which is a contradiction. It follows that (1.1) has no positive entire blow-up solutions, and the proof is completed.

Proof of Theorem 2.4. We start by showing that (1.1) has positive radial solutions. Towards this end we fix $b_{i}>a, i=1,2, \ldots, d$ and we show that the system

$$
\begin{gathered}
\phi_{i}{ }^{\prime \prime}+\frac{N-1}{r} \phi_{i}{ }^{\prime}=p_{i}(r) \phi_{i}(r) f_{i}\left(\ln \phi_{1}(r), \ln \phi_{2}(r), \ldots, \ln \phi_{d}(r)\right), \quad i=1,2, \ldots, d \\
\phi_{i}{ }^{\prime} \geq 0, \quad \text { on }[0, \infty), \phi_{i}(0)=b_{i}>a
\end{gathered}
$$

has a solution $\left(\phi_{1}, \phi_{2}, \ldots, \phi_{d}\right)$. Thus $\left(U_{1}(x), U_{2}(x), \ldots, U_{d}(x)\right)=\left(\phi_{1}(|x|), \phi_{2}(|x|), \ldots, \phi_{d}(|x|)\right)$ are positive solutions of (3.1). Integrating (3.18), for any $r \geq 0$ and $i=1,2, \ldots, d$, we have

$$
\phi_{i}(r)=b_{i}+\int_{0}^{r} t^{1-N} \int_{0}^{t} s^{N-1} p_{i}(s) \phi_{i}(s) f_{i}\left(\ln \phi_{1}(s), \ln \phi_{2}(s), \ldots, \ln \phi_{d}(s)\right) d s d t .
$$

Let $\left\{\phi_{i}^{(k)}\right\}_{k \geq 0}$ be sequences of positive continuous functions defined on $[0, \infty)$ for $i=$ $1,2, \ldots, d$ by

$$
\begin{gathered}
\phi_{i}^{(0)}(r)=b_{i} \\
\phi_{i}^{(k+1)}(r)=b_{i}+\int_{0}^{r} t^{1-N} \int_{0}^{t} s^{N-1} p_{i}(s) \phi_{i}^{(k)}(s) \\
\times f_{i}\left(\ln \phi_{1}^{(k)}(s), \ln \phi_{2}^{(k)}(s), \ldots, \ln \phi_{d}^{(k)}(s)\right) d s d t .
\end{gathered}
$$

Obviously, for all $r \geq 0$, we have $\phi_{i}^{(k)}(r) \geq b_{i}, \phi_{0} \leq \phi_{1}$. The monotonicity of $f_{i}$ yields $\phi_{1}(r) \leq$ $\phi_{2}(r), r \geq 0$. Repeating the argument, we deduce that

$$
\phi_{i}^{(k)}(r) \leq \phi_{i}^{(k+1)}(r), \quad r \geq 0, k \geq 1
$$

which means $\left\{\phi_{i}^{(k)}\right\}_{k \geq 0}$ are nondecreasing sequences on $[0, \infty)$. Since

$$
\begin{aligned}
\phi_{i}^{(k+1)^{\prime}}(r) & =r^{1-N} \int_{0}^{r} s^{N-1} p_{i}(s) \phi_{i}^{(k)}(s) f_{i}\left(\ln \phi_{1}^{(k)}(s), \ln \phi_{2}^{(k)}(s), \ldots, \ln \phi_{d}^{(k)}(s)\right) d s \\
& \leq \phi_{i}^{(k)}(r) f_{i}\left(\ln \phi_{1}^{(k)}(r), \ln \phi_{2}^{(k)}(r), \ldots, \ln \phi_{d}^{(k)}(r)\right) P_{i}^{\prime}(r),
\end{aligned}
$$


we have

$$
\begin{aligned}
\sum_{i=1}^{d} \phi_{i}^{(k+1)^{\prime}}(r) \leq & \sum_{i=1}^{d} \phi_{i}^{(k+1)}(r) \sum_{i=1}^{d} f_{i}\left(\ln \phi_{1}^{(k)}(r), \ln \phi_{2}^{(k)}(r), \ldots, \ln \phi_{d}^{(k)}(r)\right) P_{i}^{\prime}(r) \\
\leq & \sum_{i=1}^{d} \phi_{i}^{(k+1)}(r) \sum_{i=1}^{d} f_{i}\left(\ln \sum_{i=1}^{d} \phi_{i}^{(k+1)}(r), \ln \sum_{i=1}^{d} \phi_{i}^{(k+1)}(r), \ldots, \ln \sum_{i=1}^{d} \phi_{i}^{(k+1)}(r)\right) \\
& \times \sum_{i=1}^{d} P_{i}^{\prime}(r) .
\end{aligned}
$$

Let $w^{(k+1)}(r)=\sum_{i=1}^{d} \phi_{i}^{(k+1)}(r)$ which implies

$$
\frac{w^{(k+1)}(r)}{w^{(k+1)}(r) \sum_{i=1}^{d} f_{i}\left(\ln w^{(k+1)}(r), \ln w^{(k+1)}(r), \ldots, \ln w^{(k+1)}(r)\right)} \leq \sum_{i=1}^{d} P_{i}^{\prime}(r) .
$$

So, we have

$$
\int_{0}^{r} \frac{w^{\prime(k+1)}(t)}{w^{(k+1)}(t) \sum_{i=1}^{d} f_{i}\left(\ln w^{(k+1)}(t), \ln w^{(k+1)}(t), \ldots, \ln w^{(k+1)}(t)\right)} d t \leq \sum_{i=1}^{d} P_{i}(r)
$$

that is

$$
F\left(\sum_{i=1}^{d} \phi_{i}^{(k+1)}(r)\right)-F\left(\sum_{i=1}^{d} b_{i}\right) \leq \sum_{i=1}^{d} P_{i}(r), \quad \forall r \geq 0 .
$$

As $F^{-1}$ increases on $[0, \infty)$, from (3.26), we have that

$$
\sum_{i=1}^{d} \phi_{i}^{(k+1)}(r) \leq F^{-1}\left(F\left(\sum_{i=1}^{d} b_{i}\right)+\sum_{i=1}^{d} P_{i}(r)\right), \quad \forall r \geq 0 .
$$

It follows from $F(\infty)=\infty$ that $F^{-1}(\infty)=\infty$. By (3.27), the sequences $\left\{\phi_{i}^{(k)}\right\}$ are bounded and increasing on $\left[0, c_{0}\right]$ for any $c_{0}>0$. Thus, $\left\{\phi_{i}^{(k)}\right\}$ have subsequences converging uniformly to $\phi_{i}$ on $\left[0, c_{0}\right]$. Consequently, $\left(\phi_{1}, \phi_{2}, \ldots, \phi_{d}\right)$ is a positive solution of (3.18); that is, $\left(U_{1}, U_{2}, \ldots, U_{d}\right)$ is a entire positive solution of (3.1). By noticing $\phi_{i}(0)=b_{i}$ and that $b_{i} \in(0, \infty)$ was chosen arbitrarily, it follows that (1.1) has infinitely many positive entire solutions.

(i) If $P_{i}(\infty)=\infty$, since

$$
\phi_{i}(r) \geq b_{i}+b_{i} f_{i}\left(\ln b_{1}, \ln b_{2}, \ldots, \ln b_{d}\right) P_{i}(r), \quad r \geq 0,
$$


we have

$$
\lim _{r \rightarrow \infty} \phi_{i}(r)=\infty,
$$

which means that $\left(U_{1}, U_{2}, \ldots, U_{d}\right)$ are positive entire blow-up solutions of (1.1).

(ii) If $P_{i}(\infty)<\infty$, then

$$
\sum_{i=1}^{d} \phi_{i}(r) \leq F^{-1}\left(F\left(\sum_{i=1}^{d} b_{i}\right)+\sum_{i=1}^{d} P_{i}(\infty)\right)<\infty
$$

which implies that $\left(U_{1}, U_{2}, \ldots, U_{d}\right)$ are positive entire bounded solutions of (1.1). Proof of the theorem is now completed.

Proof of Theorem 2.5. If condition (2.7) holds, then we have

$$
F\left(\sum_{i=1}^{d} \phi_{i}^{(k+1)}(r)\right) \leq F\left(\sum_{i=1}^{d} b_{i}\right)+\sum_{i=1}^{d} P_{i}(r) \leq F\left(\sum_{i=1}^{d} b_{i}\right)+\sum_{i=1}^{d} P_{\mathrm{i}}(\infty)<F(\infty)<\infty .
$$

Since $F^{-1}$ is strictly increasing on $[0, \infty)$, we have

$$
\sum_{i=1}^{d} \phi_{i}^{(k+1)}(r) \leq F^{-1}\left(F\left(\sum_{i=1}^{d} b_{i}\right)+\sum_{i=1}^{d} P_{i}(\infty)\right)<\infty
$$

The last part of the proof is clear from that of Theorem 2.4. Thus we omit it.

Proof of Theorem 2.6. (i) It follows from (3.20) that

$$
\begin{aligned}
\phi_{i}^{(k)}(r) & \leq \phi_{i}^{(k+1)}(r) \leq b_{i}+\phi_{i}^{(k)}(r) f_{i}\left(\ln \phi_{1}^{(k)}(r), \ln \phi_{2}^{(k)}, \ldots, \ln \phi_{d}^{(k)}\right) P_{i}(r) \\
& \leq b_{i}+\phi_{i}^{(k)}(r) f_{i}\left(\ln \sum_{i=1}^{d} \phi_{i}^{(k)}(r), \ln \sum_{i=1}^{d} \phi_{i}^{(k)}(r), \ldots, \ln \sum_{i=1}^{d} \phi_{i}^{(k)}(r)\right) P_{i}(r) .
\end{aligned}
$$

Let $R>0$ be arbitrary. From (3.33) we get, for $k \geq 1$,

$$
\begin{aligned}
\sum_{i=1}^{d} \phi_{i}^{(k)}(R) \leq \sum_{i=1}^{d} b_{i} & +\sum_{i=1}^{d} \phi_{i}^{(k)}(R) \\
& \times \sum_{i=1}^{d} f_{i}\left(\ln \sum_{i=1}^{d} \phi_{i}^{(k)}(R), \ln \sum_{i=1}^{d} \phi_{i}^{(k)}(R), \ldots, \ln \sum_{i=1}^{d} \phi_{i}^{(k)}(R)\right) \sum_{i=1}^{d} P_{i}(R) .
\end{aligned}
$$


This implies

$$
\begin{aligned}
1 \leq & \frac{\sum_{i=1}^{d} b_{i}}{\sum_{i=1}^{d} \phi_{i}^{(k)}(R)} \\
& \quad+\sum_{i=1}^{d} f_{i}\left(\ln \sum_{i=1}^{d} \phi_{i}^{(k)}(R), \ln \sum_{i=1}^{d} \phi_{i}^{(k)}(R), \ldots, \ln \sum_{i=1}^{d} \phi_{i}^{(k)}(R)\right) \sum_{i=1}^{d} P_{i}(R) .
\end{aligned}
$$

Taking into account the monotonicity of $\left(\sum_{i=1}^{d} \phi_{i}^{(k)}(R)\right)_{k \geq 1}$, there exists

$$
L(R):=\lim _{k \rightarrow \infty}\left(\sum_{i=1}^{d} \phi_{i}^{(k)}(R)\right) .
$$

We claim that $L(R)$ is finite. Indeed, if not, we let $k \rightarrow \infty$ in (3.35), and the assumption (2.9) leads us to a contradiction. Thus $L(R)$ is finite. Since $\phi_{i}^{(k)}$ are increasing functions, it follows that the map $L:(0, \infty) \rightarrow(0, \infty)$ is nondecreasing and

$$
\sum_{i=1}^{d} \phi_{i}^{(k)}(r) \leq \sum_{i=1}^{d} \phi_{i}^{(k)}(R) \leq L(R), \quad \forall r \in[0, R], \forall k \geq 1
$$

Thus the sequences $\left\{\left(\phi_{i}^{(k)}\right)_{k \geq 1}\right\}$ are bounded from above on bounded sets. Let

$$
\phi_{i}(r):=\lim _{k \rightarrow \infty} \phi_{i}^{(k)}(r), \quad \forall r \geq 0
$$

Then $\left(\phi_{1}, \phi_{2}, \ldots, \phi_{d}\right)$ is a positive solution of (3.18).

In order to conclude the proof, it is sufficient to show that $\left(\phi_{1}, \phi_{2}, \ldots, \phi_{d}\right)$ is a blow-up solution of (3.18). Let us remark that (3.19) implies

$$
\phi_{i}(r) \geq b_{i}+b_{i} f_{i}\left(\ln b_{1}, \ln b_{2}, \ldots, \ln b_{d}\right) P_{i}(r), \quad r \geq 0 .
$$

Since $f_{i}$ are positive functions and

$$
P_{i}(\infty)=\infty,
$$

we can conclude that $\left(\phi_{1}, \phi_{2}, \ldots, \phi_{d}\right)$ is a blow-up solution of (3.18), and so $\left(U_{1}, U_{2}, \ldots, U_{d}\right)$ is a positive entire blow-up solution of (3.1). Thus any blow-up solution of (3.1) provides a positive entire blow-up solution of $(1.1)$. Since $b_{i} \in(0, \infty)$ was chosen arbitrarily, it follows that (1.1) has infinitely many positive entire blow-up solutions.

(ii) If

$$
\sup _{s \geq 0} \sum_{i=1}^{d} s f_{i}(\ln s, \ln s, \ldots, \ln s)<\infty
$$


holds, then by (3.35), we have

$$
L(R):=\lim _{k \rightarrow \infty} \sum_{i=1}^{d} \phi_{i}^{(k)}(R)<\infty
$$

Thus

$$
\sum_{i=1}^{d} \phi_{i}^{(k)}(r) \leq \sum_{i=1}^{d} \phi_{i}^{(k)}(R) \leq L(R), \quad \forall r \in[0, R], \forall k \geq 1
$$

So the sequences $\left\{\phi_{i}^{(k)}\right\}_{k \geq 1}$ are bounded from above on bounded sets. Let

$$
\phi(r):=\lim _{k \rightarrow \infty} \phi_{i}^{(k)}(r), \quad \forall r \geq 0
$$

Then $\left(\phi_{1}, \phi_{2}, \ldots, \phi_{d}\right)$ is a positive solution of (3.18).

It follows from (3.33) and (3.35) that $\left(\phi_{1}, \phi_{2}, \ldots, \phi_{d}\right)$ is bounded, which implies that (1.1) has infinitely many positive entire bounded solutions.

In the end of this work we also remark on a system with different gradient exponent

$$
\begin{gathered}
\Delta u_{1}+\left|\nabla u_{1}\right|^{a_{1}}=p_{1}(|x|) f_{1}\left(u_{1}, u_{2}, \ldots, u_{d}\right), \quad x \in R^{N}, \\
\vdots \\
\vdots \\
\Delta u_{d}+\left|\nabla u_{d}\right|^{a_{d}}=p_{d}(|x|) f_{d}\left(u_{1}, u_{2}, \ldots, u_{d}\right), \quad x \in R^{N},
\end{gathered}
$$

where $a_{i} \in(0,+\infty), a_{i} \neq 1,2, f_{i}:[0, \infty)^{d} \rightarrow[0, \infty)$ are nonnegative, continuous, and nondecreasing functions for each variable. For these cases, the problem is far more complex, and no analogous results have been established $[9,10,13,18,21]$. We also anticipate that the methods and concepts here can be extended to the systems with $q_{i}$-Laplacian as considered by Covei [14-17].

\section{References}

[1] L. Bieberbach, " $\Delta u=e^{u}$ und die automorphen Funktionen," Mathematische Annalen, vol. 77, no. 2, pp. 173-212, 1916.

[2] J. B. Keller, "On solutions of $\Delta u=f(u)$, " Communications on Pure and Applied Mathematics, vol. 10, pp. 503-510, 1957.

[3] R. Osserman, “On the inequality $\Delta u \geq f(u)$," Pacific Journal of Mathematics, vol. 7, pp. 1641-1647, 1957.

[4] C. Bandle and M. Marcus, "Large solutions of semilinear elliptic equations: existence, uniqueness and asymptotic behaviour," Journal d'Analyse Mathématique, vol. 58, pp. 9-24, 1992.

[5] A. V. Lair, "A necessary and sufficient condition for existence of large solutions to semilinear elliptic equations," Journal of Mathematical Analysis and Applications, vol. 240, no. 1, pp. 205-218, 1999. 
[6] K.-S. Cheng and W.-M. Ni, "On the structure of the conformal scalar curvature equation on $R^{n}$," Indiana University Mathematics Journal, vol. 41, no. 1, pp. 261-278, 1992.

[7] A. V. Lair and A. W. Wood, "Large solutions of semilinear elliptic problems," Nonlinear Analysis A, vol. 37, no. 6, pp. 805-812, 1999.

[8] A. V. Lair and A. W. Wood, “Large solutions of sublinear elliptic equations," Nonlinear Analysis A, vol. 39, no. 6, pp. 745-753, 2000.

[9] A. V. Lair and A. W. Wood, "Existence of entire large positive solutions of semilinear elliptic systems," Journal of Differential Equations, vol. 164, no. 2, pp. 380-394, 2000.

[10] F.-C. Şt. Cîrstea and V. D. Rădulescu, "Entire solutions blowing up at infinity for semilinear elliptic systems," Journal de Mathématiques Pures et Appliquées, vol. 81, no. 9, pp. 827-846, 2002.

[11] M. Ghergu and V. Rădulescu, "Explosive solutions of semilinear elliptic systems with gradient term," Revista de la Real Academia de Ciencias Exactas A, vol. 97, no. 3, pp. 437-445, 2003.

[12] Y. Peng and Y. Song, "Existence of entire large positive solutions of a semilinear elliptic system," Applied Mathematics and Computation, vol. 155, no. 3, pp. 687-698, 2004.

[13] X. Zhang and L. Liu, "The existence and nonexistence of entire positive solutions of semilinear elliptic systems with gradient term," Journal of Mathematical Analysis and Applications, vol. 371, no. 1, pp. 300308, 2010.

[14] D.-P. Covei, "Radial and nonradial solutions for a semilinear elliptic system of Schrödinger type," Funkcialaj Ekvacioj, vol. 54, no. 3, pp. 439-449, 2011.

[15] D.-P. Covei, “Large and entire large solution for a quasilinear problem," Nonlinear Analysis A, vol. 70, no. 4, pp. 1738-1745, 2009.

[16] D.-P. Covei, "Existence of entire radically symmetric solutions for a quasilinear system with $d$ equations," Hacettepe Journal of Mathematics and Statistics, vol. 40, no. 3, pp. 433-439, 2011.

[17] D.-P. Covei, "Schrödinger systems with a convection term for the $\left(p 1, \ldots, p_{d}\right)$-Laplacian in $R^{N}$," Electronic Journal of Differential Equations, vol. 2012, no. 67, pp. 1-13, 2012.

[18] R. Dalmasso, "Existence and uniqueness of positive solutions of semilinear elliptic systems," Nonlinear Analysis A, vol. 39, no. 5, pp. 559-568, 2000.

[19] M. Ghergu and V. D. Rădulescu, Singular Elliptic Equations: Bifurcation and Asymptotic Analysis, vol. 37 of Oxford Lecture Series in Mathematics and its Applications, Oxford University Press, Oxford, UK, 2008.

[20] S. Chen and G. Lu, "Existence and nonexistence of positive radial solutions for a class of semilinear elliptic system," Nonlinear Analysis A, vol. 38, no. 7, pp. 919-932, 1999.

[21] M. Ghergu, C. Niculescu, and V. Rădulescu, "Explosive solutions of elliptic equations with absorption and non-linear gradient term," Indian Academy of Sciences, vol. 112, no. 3, pp. 441-451, 2002.

[22] J. Serrin and H. Zou, "Existence of positive entire solutions of elliptic Hamiltonian systems," Communications in Partial Differential Equations, vol. 23, no. 3-4, pp. 577-599, 1998.

[23] A. V. Lair and A. W. Wood, "Entire solution of a singular semilinear elliptic problem," Journal of Mathematical Analysis and Applications, vol. 200, no. 2, pp. 498-505, 1996.

[24] P. Quittner, "Blow-up for semilinear parabolic equations with a gradient term," Mathematical Methods in the Applied Sciences, vol. 14, no. 6, pp. 413-417, 1991.

[25] C. Bandle and E. Giarrusso, "Boundary blow up for semilinear elliptic equations with nonlinear gradient terms," Advances in Differential Equations, vol. 1, no. 1, pp. 133-150, 1996.

[26] C. S. Yarur, "Existence of continuous and singular ground states for semilinear elliptic systems," Electronic Journal of Differential Equations, vol. 1, pp. 1-27, 1998.

[27] X. Wang and A. W. Wood, "Existence and nonexistence of entire positive solutions of semilinear elliptic systems," Journal of Mathematical Analysis and Applications, vol. 267, no. 1, pp. 361-368, 2002.

[28] X. Li, J. Zhang, S. Lai, and Y. Wu, "The sharp threshold and limiting profile of blow-up solutions for a Davey-Stewartson system," Journal of Differential Equations, vol. 250, no. 4, pp. 2197-2226, 2011.

[29] S. Lai and Y. Wu, "Global solutions and blow-up phenomena to a shallow water equation," Journal of Differential Equations, vol. 249, no. 3, pp. 693-706, 2010.

[30] X. Li, Y. Wu, and S. Lai, "A sharp threshold of blow-up for coupled nonlinear Schrödinger equations," Journal of Physics A, vol. 43, no. 16, article 165205, p. 11, 2010.

[31] J. Zhang, X. Li, and Y. H. Wu, "Remarks on the blow-up rate for critical nonlinear Schrödinger equation with harmonic potential," Applied Mathematics and Computation, vol. 208, pp. 389-396, 2009.

[32] X. Zhang, "A necessary and sufficient condition for the existence of large solutions to "mixed" type elliptic systems," Applied Mathematics Letters, vol. 25, no. 12, pp. 2359-2364, 2012.

[33] W. M. Ni, "On the elliptic equation $\Delta u=K(x) u^{(n+2) /(n-2)}=0$, its generalizations, and applications in geometry," Indiana University Mathematics Journal, vol. 31, no. 4, pp. 493-529, 1982. 


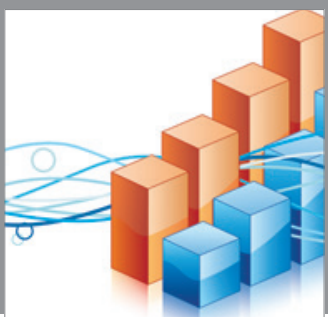

Advances in

Operations Research

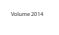

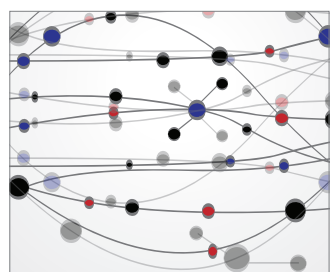

\section{The Scientific} World Journal
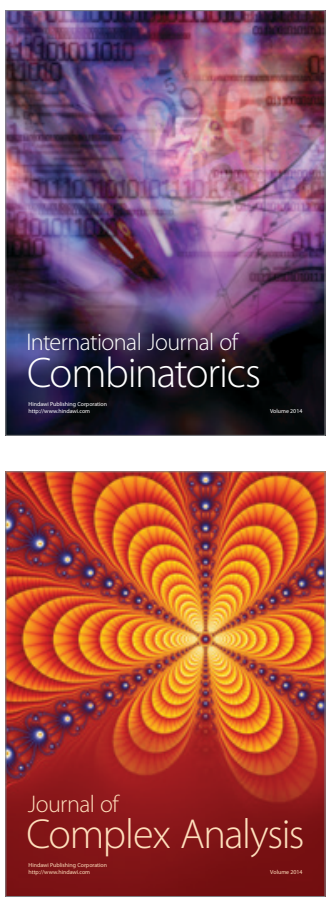

International Journal of

Mathematics and

Mathematical

Sciences
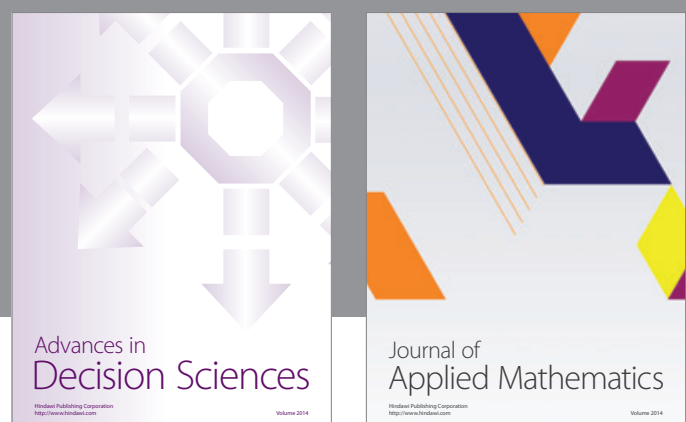

Journal of

Applied Mathematics
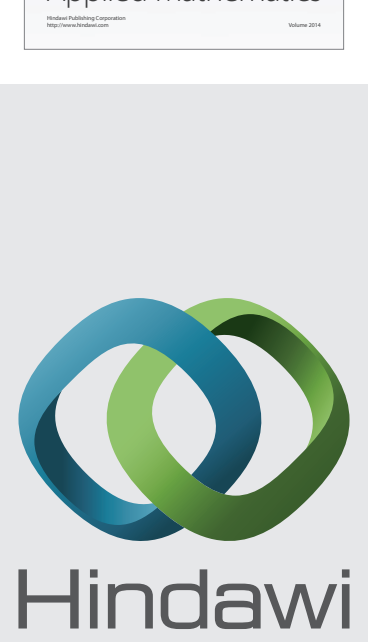

Submit your manuscripts at http://www.hindawi.com
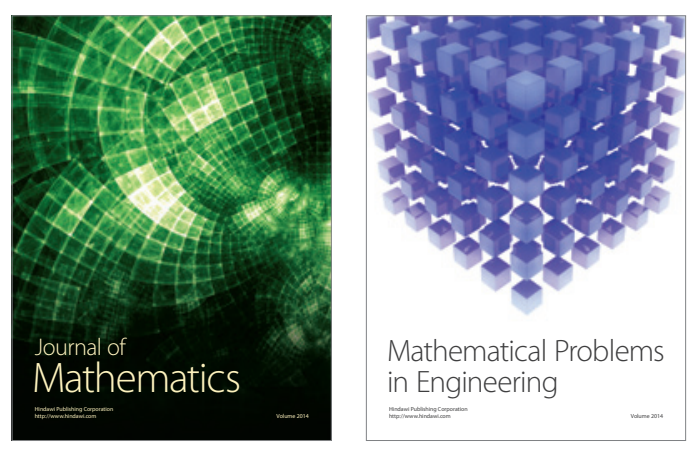

Mathematical Problems in Engineering
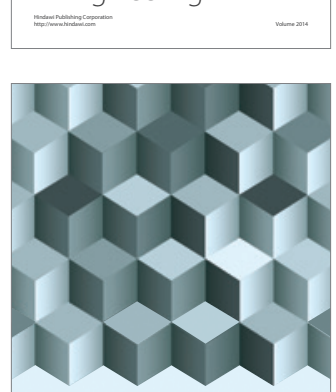

Journal of

Function Spaces
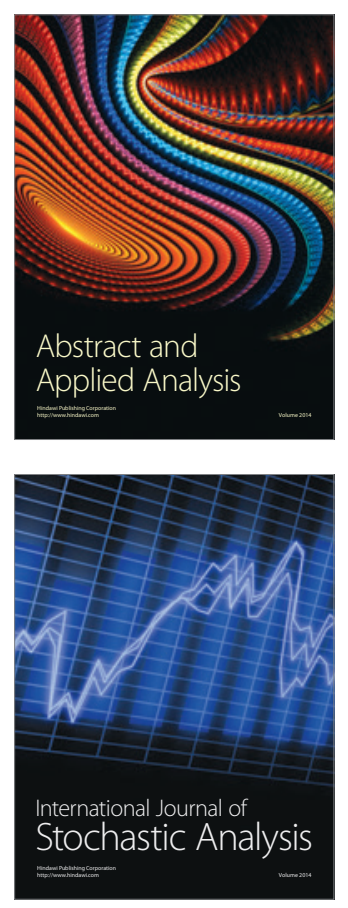

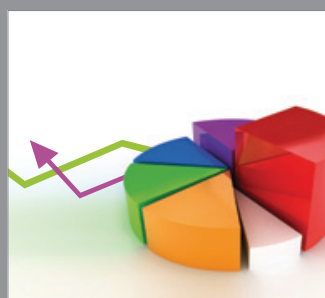

ournal of

Probability and Statistics

Promensencen
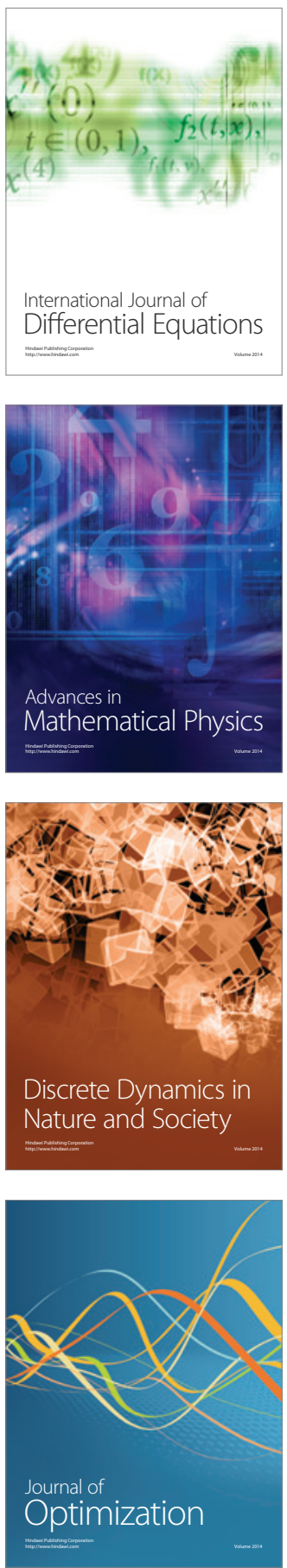